\title{
Myasthenia gravis: Association of British Neurologists' management guidelines
}

\author{
Jon Sussman, ${ }^{1}$ Maria E Farrugia, ${ }^{2}$ Paul Maddison, ${ }^{3}$ Marguerite Hill, ${ }^{4}$ \\ M Isabel Leite, ${ }^{5}$ David Hilton-Jones ${ }^{5}$
}

- Additional material is published online only. To view please visit the journal online (http://dx.doi.org/10.1136/ practneurol-2015-001126)

'Department of Neurology, Greater Manchester Neuroscience Centre, Salford, Greater Manchester, UK

${ }^{2}$ Institute of Neurological Sciences, Southern General Hospital, Glasgow, UK ${ }^{3}$ Department of Neurology, Nottingham University Hospitals NHS Trust, Queens Medical Centre, Nottingham, Nottinghamshire, UK ${ }^{4}$ Department of Neurology, Morriston Hospital, Swansea, Wales, UK

${ }^{5}$ Department of Neurology, John Radcliffe Hospital, Oxford, UK

\section{Correspondence to}

Dr Jon Sussman, Department of Neurology, Greater Manchester Neuroscience Centre, Stott Lane, Salford, Greater Manchester, M6 8HD, UK;

Jon.sussman@manchester.ac.uk

Accepted 13 April 2015

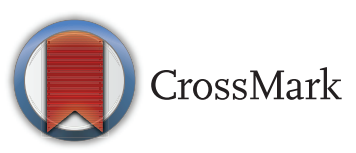

To cite: Sussman J, Farrugia ME, Maddison P, et al. Pract Neurol 2015:15:199-206.

\section{ABSTRACT}

Myasthenia gravis is an autoimmune disease of the neuromuscular junction for which many therapies were developed before the era of evidence based medicine. The basic principles of treatment are well known, however, patients continue to receive suboptimal treatment as a result of which a myasthenia gravis guidelines group was established under the aegis of The Association of British Neurologists.

These guidelines attempt to steer a path between evidence-based practice where available, and established best practice where evidence is unavailable. Where there is insufficient evidence or a choice of options, the guidelines invite the clinician to seek the opinion of a myasthenia expert. The guidelines support clinicians not just in using the right treatments in the right order, but in optimising the use of well-known therapeutic agents. Clinical practice can be audited against these guidelines.

These guidelines are designed to guide physicians and general neurologists in the management of myasthenia gravis. They are based on evidence where available, and on the experience of experts where well-established treatments lack evidence. It is not possible to consider all the potential decisions in managing myasthenia without resorting to opinion rather than evidence. ${ }^{i}$ These guidelines therefore

\footnotetext{
'There are remarkably few studies comparing treatments in myasthenia gravis. Some were established before the era of evidence-based medicine. Some pivotal positive treatment studies used only small numbers, and their flawed design may account for studies suggesting that a commonly used medication (mycophenolate mofetil) lacks efficacy. For these reasons, these guidelines provide practical advice on starting standard treatment but avoid giving authority to non-evidence-based opinion by suggesting that expert opinion is sought where evidence is lacking, such as in the choice of immunosuppressive agents.
}

direct the physicians to seek the advice of a neurologist with a specialist interest in myasthenia where the evidence base is too limited, where there is a range of treatment options or when the disease is difficult to manage.

Myasthenia gravis symptoms vary, and so patients should be managed as far as possible by one clinician. A myasthenia specialist nurse or neuromuscular advisor, if available, should be involved in the care of patients.

All patients should receive the contact details of their named clinician in case of clinical deterioration and should be aware of patient information literature available from Myaware (previously the Myasthenia Gravis Association). ${ }^{\text {ii }}$

The UK Medicines and Healthcare Products Regulatory Agency regularly updates its advice on the use of a range of medication. Please refer to contemporaneous advice on the uses and interactions of the medications discussed in these guidelines.

These guidelines can only offer suggestions on how to treat common clinical scenarios. This document is intended to offer guidance: it could be followed to the letter or used flexibly. Individuals with myasthenia vary, so it is assumed that clinicians will select therapy accordingly. ${ }^{\text {iii }}$

A completed AGREE questionnaire relevant to these guidelines is available

\footnotetext{
${ }^{\text {ii } M y a w a r e . ~ C o l l e g e ~ B u s i n e s s ~ C e n t r e, ~ U t t o x e t e r ~ N e w ~}$ Road, Derby, UK DE22 3WZ tel: +44 1332 290219. Website at http://www.myaware.org/ (accessed 23 Mar 2015).

iii The same errors in myasthenia management occur repeatedly. These include a misplaced faith in the benefits of pyridostigmine, and reluctance to use sufficient doses of prednisolone to induce remission. ${ }^{12}$
} 
on the Practical Neurology website (see online supplementary appendix).

\section{INVESTIGATIONS, INITIAL ASSESSMENT AND DIAGNOSIS}

\section{Diagnostic tests}

1. Serum anti-acetylcholine receptor (ACh-R) antibody testing: first-line investigation for non-urgent patients.

2. Thyroid function: for all patients. ${ }^{\text {iv }}$

3. Serum anti-muscle-specific kinase (MuSK) antibody testing: for all patients negative for ACh-R antibodies.

4. Neurophysiology: May help to establish the diagnosis in seronegative patients with suspected myasthenia gravis. It should be performed by a practitioner with experience of myasthenia gravis. Repetitive nerve stimulation is the initial test; if negative, consider single-fibre electromyography. ${ }^{\mathrm{V}}$ Focus the testing on symptomatic muscles.

5. MR scan of brain: Patients with negative serology and neurophysiology, and symptoms compatible with ocular myasthenia may have structural brain disease.

6. Thymus scanning: All patients with suspected myasthenia, irrespective of distribution (ocular/generalised) or serology (seropositive/negative), should undergo thymus imaging. The modality (CT or MRI) should be decided locally.

7. Referral to a myasthenia specialist: If tests are negative and myasthenia is still suspected or if a congenital myasthenia syndrome is suspected.

8. Edrophonium/Tensilon test. If there is diagnostic doubt, refer to a myasthenia gravis specialist. ${ }^{\mathrm{vi}}$

\section{Confirmation of diagnosis and subtype}

ACh-R/MuSK seropositive/seronegative
Thymoma/no thymoma
Ocular ${ }^{\text {viii//generalised }}$
Congenital: (may mimic seronegative autoimmune
myasthenia)
Possible myasthenia but tests negative: consider a muscle
disorder or refer to myasthenia expert for management.

${ }^{\text {iv }}$ Other autoimmune diseases are more common in patients with myasthenia gravis; thyroid dysfunction that may otherwise be occult may exacerbate myasthenia. $B_{12}$ deficiency is more common in patients with myasthenia gravis and may cause changes in the full blood count, including a rising mean corpuscular volume and falling white count (mimicking the effect of azathioprine). For practical management of myasthenia gravis, measuring $B_{12}$ can help. "Single-fibre electromyography lacks specificity. It helps in the clinical setting of myasthenia but may be positive in a wide range of other settings, such as denervation and myopathic disorders. It should therefore be carried out only by neurophysiologists with experience in neuromuscular transmission disorders.

${ }^{\text {vi }}$ The edrophonium test may be difficult to interpret. Conditions that mimic myasthenia may produce a positive result and there are potential cardiac complications. If serology and neurophysiology still give no diagnosis, seek a specialist opinion.

vii Thymic hyperplasia is a pathological diagnosis and cannot be evaluated by scanning. The scan is done solely to exclude thymoma.

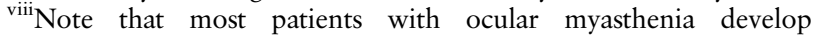
generalised myasthenia within about two years.

\section{INITIAL TREATMENT PLAN}

- Choose inpatient care: For significant bulbar symptoms early on, low vital capacity, respiratory symptoms or progressive deterioration.

- Choose outpatient care: For ocular symptoms, mild-tomoderate limb weakness and mild bulbar symptoms.

- Ocular myasthenia gravis: See section 'Treatment of ocular myasthenia gravis'.

- Generalised myasthenia gravis: See section 'Treatment of generalised myasthenia gravis'.

\section{TREATMENT OF OCULAR MYASTHENIA GRAVIS ${ }^{\mathrm{i} \times} \times$}

Starting treatment for ocular myasthenia gravis ${ }^{\mathbf{x}}$

1. Start pyridostigmine following protocol. See section 'Pyridostigmine dose escalation protocol'.

2. If the serum ACh-R antibody is positive and the patient is aged under 45 years: consider thymectomy at presentation. ${ }^{\text {xii xiii }}$

3. If symptomatic despite pyridostigmine, start prednisolone (generally given on alternate days) ${ }^{\mathrm{xiv}}$ See section 'Protocol for starting prednisolone for outpatients with ocular myasthenia gravis'.

4. If symptoms relapse on prednisolone withdrawal at a dose of $7.5-10 \mathrm{mg} /$ day (or $15-20 \mathrm{mg}$ alternate days) or greater, consider immunosuppression. ${ }^{\mathrm{xv}}$ See sections 'Patients who do not achieve remission with prednisolone' and 'Immunosuppression'.

${ }^{\mathrm{ix}}$ The recently published EFNS/ENS Guidelines ${ }^{3}$ offer identical advice.

${ }^{\mathrm{x}}$ There is little evidence for the pyridostigmine and prednisolone dose schedules recommended here; there are no studies comparing treatment regimens. These guidelines are based on the practice of experts in the field. The emphasis is on identifying the optimum dose for each patient. Undertreatment, particularly with corticosteroids, is the commonest cause of persisting symptoms.

${ }^{x i}$ Around $50 \%$ of patients with ocular myasthenia develop generalised myasthenia within 2 years and up to $75 \%$ do so within 3 years or so of disease onset. This is more likely in the seropositive group.

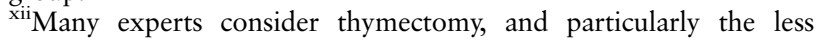
traumatic and scar-minimising video-assisted thoracoscopic thymectomy in a young person with seropositive generalised myasthenia gravis. Some experts consider video-assisted thoracoscopic thymectomy in purely ocular myasthenia gravis. Thymectomy is probably most effective if carried out early on, say 2 years after symptom onset. Thymectomy probably does not help established ocular myasthenia gravis where there is little risk of generalisation.

xiii Offering video-assisted thoracoscopic thymectomy even before starting corticosteroids may be an option for some patients. As thymectomy produces benefit slowly, this is appropriate only for patients with mild disease.

${ }^{\text {xiv }}$ An alternate day corticosteroid regimen probably reduces side effects. Daily corticosteroids may be suitable in some patients, such as those with diabetes mellitus.

${ }^{\mathrm{xv}}$ In principle, immunosuppression is only started following relapse induced by corticosteroid withdrawal. In some patients with complex medical problems, higher doses of corticosteroids may be preferable to immunosuppression, or vice versa. It is impossible to provide absolute guidance on the target corticosteroid dose. In some patients, even low-dose corticosteroids cause side effects; these might be treated with immunosuppression. Exceptional cases should be discussed with a myasthenia expert. 


\section{Pyridostigmine dose escalation protocol ${ }^{\mathrm{xvia}, \mathrm{xvib}}$}

Titrate up to find the lowest effective dose:

- Initially $30 \mathrm{mg}(1 / 2$ tablet $)$ four times daily for 2-4 days.

- Then $60 \mathrm{mg}$ (1 tablet) four times daily for 5 days and experiment with timing.

- Then increase to $90 \mathrm{mg}$ four times daily over 1 week if required. The duration of action varies, and some patients require five divided daily doses. If pyridostigmine does not control symptoms satisfactorily within a few weeks, start prednisolone.

Management of side effects: Propantheline or mebeverine can help cholinergic side effects.

Withdrawal:

- If using pyridostigmine monotherapy, prescribe the lowest effective dose.

- If asymptomatic following introduction of prednisolone only, withdraw slowly at 30-60 mg per week, until either withdrawn, or use the lowest effective dose.

\section{Protocol for starting prednisolone for outpatients with ocular myasthenia gravis ${ }^{\text {xvii }}$}

Start $5 \mathrm{mg}$ on alternate days ${ }^{\mathrm{xviii}}$ for three doses and increase by $5 \mathrm{mg}$ every three doses until symptoms improve. The maximum dose is $50 \mathrm{mg}$ on alternate days or $0.75 \mathrm{mg} / \mathrm{kg} /$ alternate day for ocular myasthenia gravis.

Clinical remission of myasthenia on corticosteroid treatment is defined as the absence of symptoms and signs after pyridostigmine withdrawal.

Some patients are resistant to corticosteroids or respond very slowly. After 3 months of treatment, non-responders should be referred to a myasthenia specialist.

Prednisolone may either induce or exacerbate diabetes mellitus. This should be monitored. ${ }^{x i x}$ Daily

\footnotetext{
${ }^{\text {xvia }}$ Use pyridostigmine carefully in patients with obstructive respiratory disease, brady-dysrhythmias, impaired renal function and recent coronary occlusion.

${ }^{\text {xvib }}$ The drug information sheet specifies that propantheline is contraindicated in myasthenia gravis: this is not correct as it is a standard treatment for the side effects of pyridostigmine.

${ }^{x v i i}$ The evidence suggests that the biggest error in managing myasthenia is the failure to induce remission in a timely manner using corticosteroids. There is a tendency to try to avoid using large doses of corticosteroids. If it is tolerated, the top dose is prednisolone $0.75 \mathrm{mg} / \mathrm{kg}$ for ocular disease. Corticosteroid-induced deterioration in myasthenia sometimes occurs. It is particularly associated with starting high-dose corticosteroids. It can occur in patients adhering to the dose regimen recommended in these guidelines, though it may also be confused with the deterioration in symptoms that continues until the benefit from corticosteroids becomes apparent, often within a week or two. If in doubt, seek expert advice.

${ }^{\text {xviii }}$ The side effects are probably minimised by using alternate day dosing.

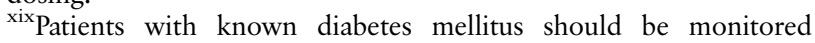
following the introduction of corticosteroids. Experts are aware of non-diabetic patients who developed diabetes mellitus, sometimes months after starting prednisolone. It is beyond the scope of these guidelines to offer specific guidance in this area. Local guidelines should be used.
}

rather than alternate day prednisolone may improve glycaemic control in patients taking treatment for elevated plasma glucose.

\section{Bone protection protocol for patients treated with corticosteroids}

Use local agreed guidelines.

\section{Prednisolone withdrawal for ocular myasthenia gravis ${ }^{\mathbf{x i}}$}

Prednisolone should be titrated down, seeking the lowest effective dose, only after achieving remission (absence of symptoms and signs having withdrawn from pyridostigmine) for at least 2-3 months:

- Reduce by $5 \mathrm{mg}$ alternate day/calendar month down to $20 \mathrm{mg}$ alternate days.

- Then reduce by $2.5 \mathrm{mg}$ alternate day/calendar month down to $10 \mathrm{mg}$ alternate days.

- Below $10 \mathrm{mg}$ alternate days reduce by $1 \mathrm{mg} /$ month. See section 'Corticosteroid maintenance dose and criteria for introducing immunosuppression' for target dose.

- Should symptoms recur, follow guidance for myasthenic relapse (see section 'Assessment and management of the relapsing patient with myasthenia).

\section{Corticosteroid maintenance dose and criteria for introducing immunosuppression}

- A prednisolone dose above 15-20 mg on alternate days is probably too high for long-term use, and is an indication to introduce immunosuppression with azathioprine. ${ }^{\mathrm{xx}}$

- Intolerable corticosteroid side effects are an indication to introduce immunosuppression to reduce the corticosteroid maintenance dose.

\section{TREATMENT OF GENERALISED MYASTHENIA GRAVIS ${ }^{\text {xxi }}$}

\section{Starting treatment for generalised myasthenia gravis}

1. Start pyridostigmine following protocol. See section 'Pyridostigmine dose escalation protocol'.

2. ACh-R antibody seropositive and aged under 45 years: consider thymectomy. ${ }^{\mathrm{xxii}}$

\footnotetext{
${ }^{\mathrm{x}}$ There is little hard evidence on the absolute safest long-term dose of corticosteroids. Since a component of the complications can be managed by bone protection, and blood pressure and lipid management, there may be individual cases with other health problems, in whom higher doses of maintenance corticosteroids would be better than immunosuppression. In principle, however, failure to control myasthenia symptoms with a 'safe' dose of corticosteroids is the main indication for starting immunosuppression.

${ }^{\mathrm{xxi}}$ There is little evidence for the pyridostigmine and prednisolone dose schedules recommended here and there have been no studies comparing treatment regimens. These guidelines are based on the practice of experts in the field. The emphasis is on identifying the optimum dose for each patient. Undertreatment, particularly with corticosteroids, is the commonest cause of persisting symptoms.

xxii Many experts consider thymectomy, particularly the less traumatic and scar-minimising video-assisted thoracoscopic thymectomy in a young person with seropositive generalised myasthenia gravis. Thymectomy appears most effective if carried
} out early on, say 2 years after symptom onset. 
3. If symptomatic despite pyridostigmine, start prednisolone (generally given on alternate days) ${ }^{x x i i i}$ See section 'Protocol for starting prednisolone for outpatients with generalised myasthenia gravis'.

4. If relapse occurs on prednisolone withdrawal at a dose of $7.5-10 \mathrm{mg} /$ day (or $15-20 \mathrm{mg}$ alternate days) or greater, introduce immunosuppression. ${ }^{\text {xxiv }}$ Immunosuppression may also be used for patients with corticosteroid-related side effects on low-dose prednisolone. ${ }^{\mathrm{xxv}}$ See sections 'Patients who do not achieve remission with prednisolone' and 'Immunosuppression'.

\section{Pyridostigmine dose escalation protocol ${ }^{\mathrm{xxvi}}$}

Titrate up to find the lowest effective dose:

- Initially $30 \mathrm{mg}$ ( $1 / 2$ tablet) four times daily for 2-4 days.

- Then $60 \mathrm{mg}$ (1 tablet) four times daily for 5 days and experiment with timing.

- Then increase to $90 \mathrm{mg}$ four times daily over 1 week if required. The duration of action varies, and some patients require five divided daily doses. If pyridostigmine fails to control symptoms satisfactorily within a few weeks, start prednisolone.

Management of side effects: propantheline ${ }^{\mathrm{xxvi}}$ or mebeverine can help cholinergic side effects.

Withdrawal:

- If using pyridostigmine monotherapy, prescribe the lowest effective dose.

- If asymptomatic after introduction of prednisolone only, withdraw slowly at 30-60 mg per week, until withdrawn, or use the lowest effective dose.

\section{Protocol for starting prednisolone for outpatients with generalised myasthenia gravis ${ }^{\mathbf{x} x i i}$}

Start $10 \mathrm{mg}$ on alternate days ${ }^{\mathrm{xxviii}}$ for three doses and increase by $10 \mathrm{mg}$ every three doses until symptoms

\footnotetext{
${ }^{x x i i i}$ An alternate day regimen is thought to reduce side effects. Daily corticosteroids may be suitable in some patients, such as those with diabetes mellitus.

${ }^{x x i v}$ In principle, other than in exceptional circumstances, immunosuppression is not started other than following relapse induced by corticosteroid withdrawal. Some patients with complex medical problems, higher doses of corticosteroids may be preferable to immunosuppression, or vice versa. Exceptional cases should be discussed with a myasthenia expert.

${ }^{\mathrm{xxv}} \mathrm{It}$ is impossible to provide absolute guidance on minimum corticosteroid requirements. There will be some patients in whom even low-dose steroids cause side effects, and who might be treated with immunosuppression. There may be good reasons why other patients are treated with a slightly higher than recommended prednisolone dose in preference to immunosuppression. Exceptions should be discussed with a myasthenia expert.

xxvi Pyridostigmine should be used carefully in patients with obstructive respiratory disease, brady-dysrhythmias, impaired renal function and recent coronary occlusion. The drug information sheet specifies that propantheline is contraindicated in myasthenia gravis: this is incorrect as it is a standard treatment for the side effects of pyridostigmine.

xxvii The evidence suggests that the biggest error in managing myasthenia is not inducing remission in a timely manner using corticosteroids. Clinicians tend to try to avoid using large doses of corticosteroids. If the patient tolerates these, the top dose is prednisolone $0.75 \mathrm{mg} / \mathrm{kg}$ for ocular disease and $1.5 \mathrm{mg} / \mathrm{kg}$ for generalised myasthenia gravis.

Corticosteroid-induced deterioration in myasthenia may sometimes occur. This can be confused with the ongoing deterioration in symptoms that continues until the corticosteroids start to help, often within a week or two. If in doubt, seek expert advice.
}

improve. Maximum dose is $100 \mathrm{mg}$ alternate days or $1.5 \mathrm{mg} / \mathrm{kg}$ for generalised myasthenia gravis. It may take many months to achieve full remission.

Remission of myasthenia on corticosteroid therapy is defined as the absence of symptoms and signs after pyridostigmine withdrawal.

For failure to respond, or side effects on increasing corticosteroids, seek expert opinion for guidance on the use of plasma exchange, intravenous immunoglobulin or immunosuppression. If not in remission after 3 months of corticosteroid treatment, refer for expert opinion.

Prednisolone may induce or exacerbate diabetes mellitus. This should be monitored. ${ }^{\text {xxix }}$ Using daily rather than alternate day prednisolone may improve glycaemic control in patients taking treatment for elevated plasma glucose.

\section{Bone protection protocol for patients treated with corticosteroids \\ Use local agreed guidelines.}

\section{Protocol for withdrawal of prednisolone for generalised myasthenia gravis ${ }^{\mathbf{x x}}$}

- Prednisolone should be titrated down seeking the lowest effective dose only after achieving remission (absence of symptoms and signs having withdrawn pyridostigmine) for at least 2-3 months.

- Reduce by $10 \mathrm{mg} /$ calendar month down to $40 \mathrm{mg}$ on alternate days.

- Then reduce by $5 \mathrm{mg} /$ calendar month down to $20 \mathrm{mg}$ on alternate days.

- Then reduce by $2.5 \mathrm{mg}$ per calendar month down to $10 \mathrm{mg}$ alternate days.

- Below $10 \mathrm{mg}$ alternate days, reduce by $1 \mathrm{mg} / \mathrm{month}$, aiming for a maintenance dose of 7 or $8 \mathrm{mg}$.

- Should symptoms recur, follow guidance for myasthenic relapse (see section 'Assessment and management of the relapsing patient with myasthenia').

\section{Corticosteroid maintenance dose and criteria for starting immunosuppression}

- A corticosteroid dose above 15-20 mg on alternate days is probably an indication to introduce alternative immunosuppression with azathioprine. ${ }^{\mathrm{xxi}}$

\footnotetext{
xxviii Alternate day dosing probably minimises side effects.

${ }^{\mathrm{xxix}}$ We recommend monitoring patients with known diabetes mellitus after starting corticosteroids. Occasionally, non-diabetic patients develop diabetes mellitus, sometimes months after starting prednisolone. It is beyond the scope of these guidelines to offer specific guidance for blood glucose monitoring; local guidelines should be used.

${ }^{\mathrm{xxx}} \mathrm{This}$ is the typical rate of corticosteroid reduction. If there are other factors, such as serious side effects, the corticosteroids might be withdrawn more rapidly. Discuss with a myasthenia expert.

${ }^{x x x i}$ There is little hard evidence on the absolute safest long-term dose of steroids. Since a component of the complications can be managed by bone protection, and blood pressure and lipid management, there may be individual cases with other health problems, in whom higher doses of maintenance steroids would be preferable to immunosuppression. In principle, however, failure to control myasthenia symptoms with a 'safe' dose of corticosteroids is the primary indication for the initiation of immunosuppression.
} 
- Intolerable corticosteroid side effects are an indication to introduce immunosuppression to reduce the corticosteroid maintenance dose.

\section{PATIENTS WHO DO NOT ACHIEVE REMISSION WITH PREDNISOLONE}

Patients who do not achieve symptom remission on the prednisolone escalation protocol

- Some patients appear to fail to respond to the recommended doses of prednisolone. This may result from a slow response to corticosteroids, which may take up to 3 months and sometimes much longer.

- The commonest reason for a patient to fail to respond to corticosteroids is using an insufficient dose. ${ }^{\text {xxxii }}$

- If a patient does not respond adequately to the target dose after 3 months, seek a specialist opinion.

\section{IMMUNOSUPPRESSION}

\section{Corticosteroid maintenance dose and criteria for} introducing immunosuppression

- We recommend that an immunosuppressive agent is introduced only if a patient does not achieve remission on corticosteroid monotherapy. ${ }^{\text {xxxiii }}$

- A corticosteroid dose above 15-20 mg on alternate days is probably unacceptable for long-term use. ${ }^{\text {xxiv }}$

- Intolerable corticosteroid side effects are an indication to introduce immunosuppression to reduce the corticosteroid maintenance dose.

- Immunosuppression should be considered early on in patients with diabetes mellitus, osteoporosis, or ischaemic heart disease or significant bulbar or respiratory weakness that does not respond rapidly to corticosteroids.

- Immunosuppression and vaccination: Vaccines are less effective in immunosuppressed patients. Live vaccines are absolutely contraindicated and there is an increased risk from live-attenuated vaccines. Patients requiring immunosuppression should have their vaccinations updated. This might include pneumococcus, influenza, Haemophilus influenzae and Varicella zoster. ${ }^{\mathrm{xxxv}}$ Where feasible, clinicians should plan vaccinations (with blood tests to assess response) before starting immunosuppression.

\footnotetext{
${ }^{x x x i i}$ A good source of ideas if things do not proceed according to plan is Hilton-Jones. ${ }^{2}$

xxxiii The pivotal paper that showed the corticosteroid-sparing effect of azathioprine ${ }^{4}$ has been interpreted as suggesting that all patients require immunosuppression to achieve a safe maintenance dose of prednisolone. However, many patients who achieve remission can be maintained on low-dose prednisolone without needing immunosuppression. There is no evidence-based way to identify patients who will fail to achieve remission on corticosteroid monotherapy, so it should be attempted in all.

${ }^{x x x i v}$ There is little hard evidence on the absolute safest long-term dose of corticosteroids. Since a component of the complications can be managed by bone protection, and blood pressure and lipid management, there may be individual cases with other health problems, in whom higher doses of maintenance steroids would be preferable to immunosuppression. In principle. however, failure to control myasthenia symptoms with a 'safe' dose of corticosteroids is the primary indication for the initiation of immunosuppression.

${ }^{x x x v}$ Local or national guidelines should be followed.
}

- Corticosteroids in high doses can reduce the immune response to vaccines and live virus vaccinations should be delayed for at least 1 month after stopping high-dose corticosteroids.

\section{Immunosuppression with azathioprine ${ }^{\mathbf{x x x v i}}$}

- Azathioprine is the first-line agent. Thiopurine methyltransferase (TMPT) should be measured in view of the association between TMPT deficiency and myelosuppression with azathioprine. TMPT deficiency is not a contraindication to using azathioprine but may reduce the dose required. ${ }^{\text {xxvii }}$ Patients with very low TMPT activity should not take azathioprine.

- Azathioprine should be increased over 1 month to a maintenance dose of $2.5 \mathrm{mg} / \mathrm{kg}$. Blood tests should be monitored weekly (full blood count, urea and electrolytes, liver function tests) as the dose is titrated upwards. ${ }^{\text {xxxiii }}$ The dose may need to be modified in light of side effects or changes in blood tests. We recommend using local shared-care protocols.

- Azathioprine is slow to achieve maximum effect. ${ }^{\text {xxxix }}$ The target is to achieve a maintenance dose of prednisolone of below $20 \mathrm{mg}$ on alternate days after 2 years. Failure to achieve efficacy may be a consequence of inadequate dosing. Not all patients respond to azathioprine. Corticosteroids should be withdrawn using the protocol described. $^{\mathrm{xl}}$

${ }^{x x x v i}$ There is a range of immunosuppressive agents that can be successfully used in myasthenia despite the lack of robust evidence for their use. All studies are underpowered or suffer from design faults. The criteria for selecting agents might include safety in pregnancy, time to onset of efficacy and risk of complications, although there is often a lack of evidence in these areas. In practice, we recommend that non-experts manage patients with corticosteroids to induce remission, and use azathioprine as a corticosteroid-sparing agent. If this fails, then the patient should be referred to a myasthenia expert to advise on alternative immunosuppressive agents.

xxxvii Note that TMPT levels will be modified in patients who have received blood products. Patients with low serum TMPT concentrations can be treated with lower doses of azathioprine. Some experts recommend using a dose of $25 \mathrm{mg}$ once daily. In view of the evidence from other conditions that correlates efficacy with a rise in mean corpuscular volume and/or a drop in peripheral lymphocyte count, others titrate the dose up until there is a haematological response. 6-thioguanine nucleotides (6-TGN), which are incorporated into DNA, are the active metabolites of azathioprine. 6-TGN has started to be used to monitor compliance and toxicity. It is not widely available but can be used to guide dosing.

xxxviii In rheumatological and gastroenterology patients, azathioprine efficacy correlates with a drop in lymphocyte count and/or an elevation in mean corpuscular volume. In inflammatory bowel disease, macrocytosis appears to be the better marker. There is no evidence in myasthenia, but anecdotal observations suggest that a failure to respond to azathioprine is more common in patients without a change in these indices. We recommend treating using the initial $\mathrm{mg} / \mathrm{kg}$ dosing, and following local shared-care protocols. A specialist opinion should be sought if difficulties arise.

${ }^{x x x i x}$ The pivotal azathioprine study ${ }^{4}$ showed efficacy at 2 years but not at 1 year. It is likely that this varies between patients.

${ }^{\mathrm{xl}} \mathrm{A}$ patient taking $100 \mathrm{mg}$ alternate day prednisolone would take 14 months to reduce to a safe maintenance dose. 
- There is a debate as to whether taking azathioprine is a contraindication to using intrauterine contraceptive devices. This is based on a few case reports. There is probably no good reason to avoid intrauterine contraceptive devices, but specialist advice should be sought. It is possible to take azathioprine during pregnancy, ${ }^{x l i}$ and it is considered safe for breastfeeding mothers. ${ }^{\text {xlii }}$

- Myasthenia gravis may relapse on corticosteroid withdrawal. This may be a consequence of a slow response to azathioprine or using too low a dose. The lowest effective corticosteroid dose before relapse is the maintenance dose. If this is above $20 \mathrm{mg}$ on alternate days, this is considered a relapse and requires action. See section 'Assessment and management of the relapsing patient with myasthenia'.

\section{Other immunosuppressive agents}

Mycophenolate mofetil, methotrexate, ciclosporin, rituximab

These agents have a role in managing poorly responsive myasthenia when azathioprine has failed or the patient cannot tolerate it. Poor study design and small numbers means that the literature is unhelpful. Most patients should respond to pyridostigmine, prednisolone and azathioprine if used correctly. Non-responders or those with treatment complications should be referred to a myasthenia specialist. Selecting a second-line agent must take into account factors such as concurrent renal disease and reproductive intentions; ciclosporin appears no more harmful than azathioprine in pregnancy. Methotrexate is teratogenic in men and women, and effective contraception is mandatory until at least 3 months after stopping the drug. Mycophenolate mofetil may cause congenital malformations and so women should use effective contraception during its use and for at least 6 weeks after stopping it.

\footnotetext{
${ }^{x l i}$ Azathioprine is widely considered to be low risk in pregnancy and is the drug of choice in the UK. In the USA, it is considered high risk. This differing advice is based on a small number of animal studies and case reports. Other immunosuppressive agents pose more definite risks to the fetus. Studies, largely from patients with inflammatory bowel disease taking azathioprine, suggest a modestly increased risk of low birth weight or atrial or ventricular septal defects, though other studies suggest that these relate to disease activity rather than to azathioprine. A detailed discussion of these issues is beyond the scope of these guidelines. The risks and benefits should be discussed with individual patients.

xlii Azathioprine is either undetectable in breast milk or is found in very low concentrations. The only concern would be that higher concentrations may occur in the breast milk of mothers with a low serum TMPT concentration. Asymptomatic low levels of neutrophils may develop in babies, leading some experts to recommend monitoring exclusively breastfed babies, though most feel that this is unnecessary. The concentration of azathioprine in breast milk drops significantly $4-6 \mathrm{~h}$ after taking the drug. A detailed discussion of these issues is beyond the scope of these guidelines.
}

\section{ASSESSMENT AND MANAGEMENT OF THE RELAPSING PATIENT WITH MYASTHENIA}

Determine whether the patient's deterioration is a myasthenic relapse, a side effect of treatment or another condition? xliii $^{\text {a }}$

Is the deterioration due to intercurrent infection (the most common cause of relapse, after drug reduction)?

Has the patient received medication that causes myasthenic weakness? (eg, antibiotics, betablockers)? ${ }^{\text {xliv }}$

Is the deterioration a consequence of changes in treatment, particularly steroid withdrawal?

- If a cause for myasthenia deterioration can be found, it should be managed.

- Patients with myasthenia deterioration should return to the steroid increase protocol, increasing prednisolone until symptoms are controlled up to the target dose of $1.5 \mathrm{mg} / \mathrm{kg}$ or $100 \mathrm{mg}$ alternate day for generalised myasthenia and $0.75 \mathrm{mg} / \mathrm{kg}$ or $50 \mathrm{mg}$ alternate days for ocular myasthenia. ${ }^{x l v}$

- If the relapse occurred on a dose of prednisolone above $15-20 \mathrm{mg}$ alternate days in a patient treated without receiving azathioprine, then azathioprine should be introduced following the protocol described above.

- If the myasthenia deterioration was a result of steroid withdrawal in a patient receiving azathioprine, the relapse may have been a result of too short a duration of

\footnotetext{
xliii A comprehensive list of the causes of weakness is beyond the scope of these guidelines. Patients may describe tiredness, which may be hard to distinguish from fatigable muscular weakness. Steroid-induced muscle weakness is recognised. Neurological deterioration is common in patients with intercurrent infection. Other autoimmune conditions are more common in myasthenia, such as thyroid disorders and B12 deficiency, and may cause neurological symptoms. Degenerative spine disease resulting in myelopathy or lumbar canal stenosis may give rise to weakness. In most cases, the symptoms and signs are inconsistent with myasthenia and careful history and examination with targeted investigations should reveal the diagnosis. If in doubt, a neurological opinion should be sought or an expert in myasthenia should be asked to review.

${ }^{x l i v} \mathrm{~A}$ range of medications may cause myasthenia or result in increased weakness in patients known to have myasthenia. Certain antibiotics are particularly implicated, such as aminoglycosides. Some medications are well recognised as having this effect while other agents are described in case reports. Clinical decisions must depend on circumstances. A list of medications can be found on the UK-based Myasthenia Gravis Association website (http://www.myaware.org/information-for-medical-professionals/39xii-contraindications-of-drugs-that-can-make-myasthenia-worse). A more detailed review can be found on the Myasthenia Gravis Foundation of America website. (http://www.myasthenia.org/ LinkClick.aspx? fileticket $=J u F v Z P P q 2 v g \% 3 D$ ).

${ }^{x l v}$ Sometimes, especially when a patient is slowly withdrawing prednisolone at the rate of $1 \mathrm{mg}$ per month, the reversal of one or two of the previous reductions will bring the myasthenia under control. If not, revert to the initial steroid upward titration protocol.
} 
azathioprine for maximum efficacy, or too low a dose. The prednisolone should then be titrated up following the protocol until symptomatic control is regained. Following the protocol, the prednisolone should then be titrated down to the lowest effective dose. If this is greater than $20 \mathrm{mg}$ alternate days, a specialist opinion should be sought.

\section{INPATIENT MANAGEMENT OF MYASTHENIA GRAVIS}

\section{General principles}

- A patient should be managed in hospital for significant bulbar symptoms, low vital capacity, respiratory symptoms or progressive deterioration.

- Assessment by speech and language therapist to advise on swallowing is mandatory.

- Regular assessment of vital capacity is mandatory.

\section{Intravenous immunoglobulin use for severe bulbar or respiratory symptoms ${ }^{\text {xlvi }}$}

- 1-2 g/kg is the total dose.

- This may be given as $0.5 \mathrm{~g} /$ day for 2 days, or $0.4 \mathrm{~g} /$ day for 5 days. Follow local practice. ${ }^{\text {xlvii }}$

- If symptoms persist despite the use of intravenous immunoglobulin, request specialist opinion on whether to use a second dose of intravenous immunoglobulin or to use plasma exchange. ${ }^{\text {xlviii }}$

\section{Plasma exchange}

- May be preferred in the presence of specific risk factors for intravenous immunoglobulin.

\section{Prednisolone}

- Start by following the standard protocol for generalised myasthenia gravis. See section 'Protocol for starting prednisolone for outpatients with generalised myasthenia gravis'.

\section{MANAGEMENT OF MYASTHENIA GRAVIS IN INTENSIVE CARE UNITS}

Patients may require intensive care or highdependency care to monitor respiratory function.

\footnotetext{
${ }^{\mathrm{xlvi}}$ In the presence of limb symptoms only, treatment should be with corticosteroids in the first instance. These can be slow to take effect, during which time the patient may continue to deteriorate. This should not be confused with corticosteroid-induced deterioration. Intravenous immunoglobulin has rare but life-threatening side effects and should not be used as first-line agent for all myasthenia patients. Corticosteroids are the primary treatment for moderate or severe myasthenia. Note: the duration of efficacy of intravenous immunoglobulin is 3-4 weeks, after which a stable patient may deteriorate if the patient is not established on an effective corticosteroid dose.

xlvii Intravenous immunoglobulin increases blood viscosity. Patients with known vascular occlusive disease might be given this over more rather than fewer days. Careful hydration might be a sensible precaution.

xlviii We prefer intravenous immunoglobulin as the treatment of choice as it is frequently easier and faster to give. Plasma exchange may be better if there are specific immunoglobulin risk factors (thrombotic tendency, unstable angina, recent stroke, IgA deficiency, etc).
}

For ventilated patients:

- Start prednisolone at $100 \mathrm{mg}$ alternate days.

- Start intravenous immunoglobulin immediately.

- Avoid inappropriate use of intravenous magnesium. ${ }^{\text {xlix }}$

- Consider avoiding pyridostigmine/neostigmine as these may increase secretions.

For non-ventilated patients:

- Start prednisolone following the standard protocol for generalised myasthenia gravis.

- Start intravenous immunoglobulin immediately.

- Avoid using pyridostigmine/neostigmine.

\section{MYASTHENIA GRAVIS IN PREGNANCY'}

- It is important to plan well in advance of pregnancy to allow time to optimise myasthenic status, thyroid function and the drug regimen.

- Use multidisciplinary liaison throughout the pregnancy, delivery and neonatal period.

- Provided that the myasthenia gravis is under good control before a pregnancy, most women can be reassured that it will remain stable throughout pregnancy and the postpartum months.

- The aim should be for spontaneous vaginal delivery (and actively encouraged).

- Those with severe myasthenic weakness need careful multidisciplinary management with prompt access to specialist advice and facilities.

- Newborn babies of myasthenic mothers risk transient myasthenic weakness (with onset up to a few days postpartum) even if the mother is well controlled. These babies should have access to neonatal high-dependency support.

- Fetal arthrogryposis is a rare but recognised complication of maternal myasthenia gravis.

- Pyridostigmine is not expected to cross the placenta, and there have been no reports of fetal malformations. It is compatible with breast feeding.

- Prednisolone has not been shown to increase fetal malformations.

- Azathioprine appears safe in pregnancy, as is ciclosporin.

- Mycophenolate mofetil and methotrexate are not safe in pregnancy and methotrexate is not safe in breast feeding.

\section{NOTES}

\section{Thymectomy for thymoma}

Refer to thoracic surgeon experienced in this procedure, with the support of an appropriately skilled anaesthetist in a hospital with immediate access to a neurology team who have experience in myasthenia, because of the risk of perioperative complications. Patients should achieve

\footnotetext{
${ }^{x l i x}$ Note that antacids, laxatives and supplements may contain magnesium.

${ }^{1}$ A UK workshop in May 2011 discussed the practical clinical management issues relating to pregnancy in women with myasthenia gravis; this brief summary is based on its conclusions. For more detail, see Norwood et al. ${ }^{5}$
} 
optimum control of myasthenia before thymectomy, even if this means delaying surgery.

\section{Thymectomy for in patients without thymomali}

Thymectomy is a reasonable treatment option for patients who

- are aged $<45$ years

- have positive serum anti-ACh-R antibody

It may be more effective if carried out early, so should be discussed early following diagnosis.

Patients should achieve optimum control of myasthenia before elective thymectomy.

Refer to thoracic surgeon experienced in this procedure, with the support of an appropriately skilled anaesthetist in a hospital with immediate access to a neurology team with experience in myasthenia, because of the risk of perioperative complications.

Thymectomy may induce remission, may prevent generalisation of ocular myasthenia and may reduce corticosteroid requirements. ${ }^{\text {lii }}$ Endoscopic thymectomy has improved the cosmetic outcome. A specialist in myasthenia could offer guidance and only a thoracic surgeon experience in this procedure should carry out the procedure.

\footnotetext{
${ }^{\mathrm{li}}$ Thymectomy for myasthenia in patients without thymoma is currently the subject of an international multicentre study; the results will be available after 2015. The trial examines thymectomy by sternotomy (not by video-assisted thoracoscopic thymectomy). Thymectomy may induce remission in some patients and reduce treatment requirements in others. Current recommendations should be followed.

lii Thymectomy has not been subject to assessment in modern studies; however, the benefits listed would be expected. The current thymectomy study will determine the steroid-sparing benefits of thymectomy
}

\section{Criteria for using intravenous immunoglobulin}

Intravenous immunoglobulin should be used only for significant myasthenia symptoms in the inpatient setting. It should not generally be used as a maintenance therapy. Patients whose myasthenia symptoms cannot be controlled without more than one course of intravenous immunoglobulin should have an expert opinion.

Acknowledgements We would like to thank the members of the Association of British Neurologists for reviewing a late version of the draft and suggesting modifications.

Contributors JS: idea for the guidelines and editing manuscript. All contributors attended initial guideline drafting meeting. All authors contributed to modifying guidelines until final version achieved.

Competing interests None declared.

Provenance and peer review Commissioned. Externally peer reviewed. This paper was reviewed by Jon Walters, Swansea, UK.

\section{REFERENCES}

1 Jacob S, Viegas S, Lashley D, et al. The Bare Essentials: Myasthenia gravis and other neuromuscular junction disorders. Pract Neurol 2009;9:364-71.

2 Hilton-Jones D. What to do when... The patient fails to respond to treatment: myasthenia gravis. Pract Neurol 2007;7:405-11.

3 Elsais A, Argov Z, Evoli A, et al. EFNS/ENS Guidelines for the treatment of ocular myasthenia. Eur J Neurol 2014;21:687-93.

4 Palace J, Newsom-Davis J, Lecky B. A randomised double-blind trial of prednisolone alone or with azathioprine in myasthenia gravis. Neurology 1998;50:1778-83.

5 Norwood F, Dhanjal M, Hill M, et al. Myasthenia in pregnancy: best practice guidelines from a UK multispecialty working group. J Neurol Neurosurg Psychiatry 2014;85:538-43. 